\title{
A SHIP NETWORK DYNAMIC ROUTING ALGORITHM BASED ON VECTOR NETWORK
}

\author{
Jianguang Zhao', 2 \\ Jingjing Fan ${ }^{1}$ \\ ${ }^{1}$ Institute of Information Science, Beijing Jiaotong University, Beijing, China \\ ${ }^{2}$ Information Engineering Institute, Hebei Institute of Architectural and Civil Engineering, Zhangjiakou, Hebei, China
}

\begin{abstract}
In view of the characteristics of low speed mobile ship network, strong network dynamics and large signal interference, this paper proposes a low-rate mobile sensor network routing algorithm. In mobile wireless sensor networks, network topology changes frequently is a new challenge for resource constrained sensor node. Constantly updated network topology, sensor nodes need to exchange data frequently in order to maintain fresh route, resulting in sensor nodes consume a lot of energy. To solve this problem, to reduce control overhead is considered to be an effctive strategy. In this paper, we present a vector network-based dynamic routes (VNDR) scheme aims to minimize the control cost of the sensor nodes. We propose data exchange based on binary-coded vector address to reduce the control cost, moreover, we present a set of communication rules that govern the routes search procedure thereby requiring little cost of packet analysis to determine the next hop of node. VNDR performance was compared with well known scheme such as SPIN and DD. Simulation results show that, our VNDR scheme reduced the network control overhead and improved network lifetime.
\end{abstract}

Keywords: Ship network; Vector Network; Vector address; Routes discovery; Wireless Sensor Network

\section{INTRODUCTION}

In the "13th Five-Year Plan", the state explicitly proposed the development of the marine economy. Ocean communication is also an important and crucial technology in the livelihood of the people and marine militaries. It is also a key discipline developed and paid attention by the state in recent years. It is also the goal that the marine science and technology circles have been pursuing for many years. Wireless sensor networks are widely used in the fields of reconnaissance, environmental monitoring and target location. They can sense, collect and process the object information within the network coverage in real time and send them to the observer. It has the advantages of wide coverage, remote monitoring, high monitoring accuracy, fast and lowcost network.

Wireless Sensor Network(WSN) - a self-organized network of smart dust which has limited computing and storage power, has widely used in Military areas and production and living areas [1]. In WSN, there is an enormous number of sensor nodes are deployed to collect, process and forwarding sensing data with wireless channels. [2] These battery-powered sensor nodes worked cooperatively to monitor and report the interesting information to base station or sink. In order to reduce energy consumption and prolong network lifetime, various routing protocols had proposed so far. Most of these routing protocols assumed sensor nodes to be immobile in the WSN implementation. However, there are applications environments naturally require mobile node to complete the monitoring mission, [1] e.g., in a individual combat system, a battlegroup need search forward holding formation. Therefore everyone need communication with each other to improve battlegroup manoeuvrable. Each soldier moves with low-speed so as not to expose themselves to be discovered by the enemy. Similary, in an Intelligent Transport System(ITS), a lot of immobile sensor nodes are deployed at car park, rockfall area, and traffic entrance as ITS infrastructure. In contrast, there are numerous moving vehicle in ITS. Some scholars named it Internet of Vehicles, which is constituted by mobile nodes, Vehicle position, speed, and infrastructure. 
Because of node mobility, the network topology changes frequently, it brings new challenges for data transmission and routing protocols. Hence, network topology need to constantly updata, leading to frequent data transmission and more energy consumption.So far, routing protocols have been proposed are most based on immobile, these ones not suitable for mobile scenario. To cope with the dynamic network topology, new routing scheme should be presented to accommodate the mobility of nodes.

In this paper, a novel scheme called Vector Network-Based Dynamic Routes(VNDR) is proposed for low-speed mobility WSN. The scheme is suitable for small scale low-speed and high real-time requirements scenario. The proposed scheme enables nodes to maintain optimal multiple routes with each other. Each node in the network has a unique ID, which by binary encoding to form vector address. Moreover, packet transmission scheme using multi-hop transmission, route information contained in the data packet. Thus, when a node receives a packet, from the vector cut a sub-vector to determine next hop. It aims to optimize transmission delay and energy consumption and quality of service performance using vector address. Simulation results reveal decreased transmission delay, control data overhead and energy consumption of VNDR compared to other state-of-the art.

The rest of this paper is organized as follows: Part II describes the related work about mobile WSN. Part III presents VNDR routing mechanism. To evaluate the new proposed scheme, simulation and performance compare are presented in Part IV. Section V concludes the pater, finally.

\section{RELATED WORK}

Several schemes have been proposed for mobile WSN in the last decade. Abdul et al. [1] presented a Virtual Grid-Based Dynamic Routes Adjustment Scheme for Mobile Sink-Based Wireless Sensor Networks(VGDRA) that constructs a virtual grid aim to minimize the routes reconstruction overhead and balance the nodes energy dissipation. In this scheme, a mobile sink moving around the border-line of sensor field. However, nodes along the border line spents high energy consumption thereby causing hot-spot problem. Yet another similar approach can be found in [11], which places a application of WSN-based smart grid(WSN-SG).

B.B et al. [6] proposed a new clustering algorithm Distributed load balancing Unequal Clustering in wirelesssensor networks using Fuzzy approach (DUCF) in that make use of fuzzy logic to elect cluster head $(\mathrm{CH})$. DUCF although improves the network lifetime, however, it employs residual energy, node degree and distance to BS as fuzzy input variables, and chance and size as fuzzy output variables. Due to less number of fuzzy inference parameters, it can not accurately reflect the real network. An Efficient Multipath Dynamic Routing Protocol for Mobile WSNs (M-DART) [2] which constructs multipath routing protocols for Mobile WSN by Distributed Hash Tables (DHTs).A similar approach has also been proposed in Optimizing LEACH clustering algorithm with mobile sink and rendezvous nodes (LEAH-SR)[10] which constructs a mobile sink(MS) as a way to reduce energy consumption and a rendezvous node( $\mathrm{RN})$ to act as a store node. Proactive Highly Ambulatory Sensor Routing (PHASeR) protocol for mobile wireless sensor networks proposed by T.H and F.H in [12], proactively constructs a hop-count metric to implement dynamic and robust routing in mobile WSN.

Tab.1 presents a performance comparison of the variously discussed schemes. We evaluated each scenario by comparing parameters mobile node, delay, complexity and simulation tools. The mobile node specifies the type of mobile node, including ordinary nodes and sink. The second parameter provides an estimate of the network delay involved in communicating and holding routing updates. Similarly, the complexity is an estimate the difficulty of implementation of the scheme. Finally, the last parameter indicate simulation tools used in each paper.

Based on the above literature, it is observed that the mobile node brings topology update speed up and network dalay increase. Though these related works has been improved the performance of mobile WSN, still no scenario is discovered to reduce the network delay and conctrol overhead, simultaneously, prolongs network lifetime. The main contribution of this paper is reducing the network control data overhead by encoding node ID to vector address. It offers a novel solution for route discovery without too many constrains for real motes. Compared to existing schemes, the VNDR is a viable choice in mobile WSN.

Tab. 1. Performance comparison of discussed schemes.

\begin{tabular}{|c|c|c|c|c|}
\hline Scheme & $\begin{array}{c}\text { Mobile } \\
\text { Node }\end{array}$ & Delay & $\begin{array}{c}\text { Comple- } \\
\text { xity }\end{array}$ & $\begin{array}{c}\text { Simulation } \\
\text { Tools }\end{array}$ \\
\hline VGDRA [1] & Sink & Moderate & Low & NS-2 \\
\hline WSN-SG [11] & Sink & High & Low & No \\
\hline DUCF [6] & None & High & High & MATLAB \\
\hline M-DART [2] & All & High & High & NS-2 \\
\hline LEAH-SR [10] & Sink & Moderate & Low & MATLAB \\
\hline PHASeR [12] & All & High & High & OPNET \\
\hline
\end{tabular}

\section{THE PROPOSED SCHEME}

In this section, we detail our proposed solution, including how to encode vector address and how to discover route by cutting vector address. We design a new network layer architecture including data palne, control plane and management plane. The main innovation in the data plane and some work about control plane, management plane inherited the art.

This mechanism is divided into two stages: the route discovery phase and data forwarding phase as show in Fig. 1. The mission of route dicovery phase is to complete the routing updata, the stage is divided into route acquisition phase and route filtering pahse. Furthermore, the data forwarding phase is a data communication phase, the minimum length of vector addresses is prefered to reduce traffic and delays. 
Fig. 1. is Operational diagram of VNDR.

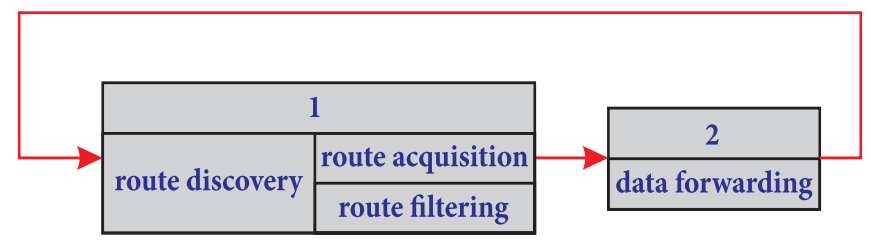

Fig. 1. Operational diagram of VNDR

\section{NETWORK FEATURES}

It is necessary to highlight the assumptions of the mobile WSN, before a detailed discussion VNDR scheme. Some of the main features of VNDR are as follows:

a. The number of nodes is not too much, and all nodes are of homogeneous architecture.

b. Nodes are randomly deployed and all nodes moving at a lower speed.

c. All nodes move throughout in its communication radius.

d. The sensor field is not too large.

e. The scheme is suitable for high real-time requirements scene, and has a weak quality of service.

\section{THE VECTOR ADDRESS CONSTRUCTION}

The VNDR scheme constructs the Vector Address (VA) by encoding all branches of any node which called Virtual Port Number (VPN). VA is an swiching address based on the VPN of nodes. The input and output port numbers (I/OPN) numbered from 1started, called Virtual Port Number (VPN). The communication path information included in the packet of $\mathrm{VN}$. The path information is a sequence of virtual port numbers, each node on the path corresponding to a virtual port number in sequence. The next-hop is determined by output virtual port number of nodes on the route. Network topology as shown in Fig. 2., virtual port number were constructed by each node according to the network topology.

Fig. 2. is an example of virtual port number were constructed.

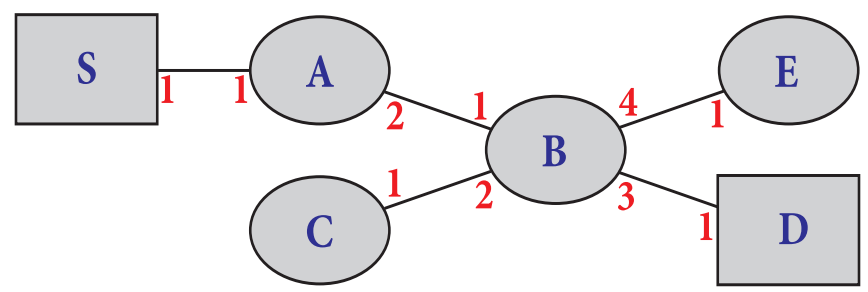

Fig. 2. An example of virtual port number were constructed

Each node builds the virtual port number depending on the degree of a node, take A for example, the degree of A is 2, similily, the degree of $B$ is 4 . Therefore, based on the above assumptions, we can build the vector address. Vector Addresses are divided into two types: Forward Vector Address(FVA) and back Backward Vector Address(BVA).Easy to deduce, Forward Vector Address(route) from the source node $S$ to the destination node $D$ VSD $=123=\{1,2,3\}$. Fig. 3 . shows an example of the construction for Vector address $\mathrm{V}_{\mathrm{SD}}$.
Fig. 3. is an example of construction for Vector address $V_{S D}$.

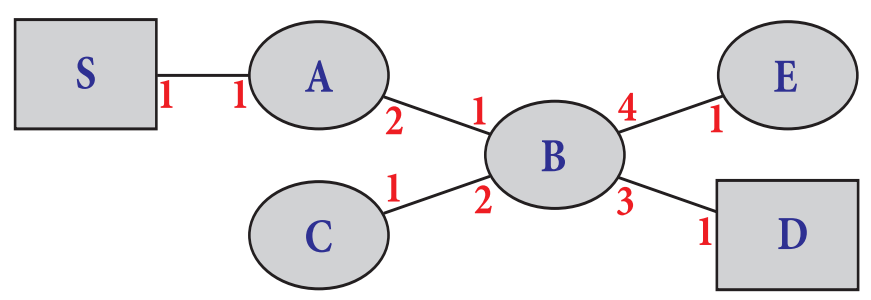

Fig. 3. An example of construction for Vector address VSD

Similarly, $\mathrm{V}_{\mathrm{CE}}=14, \mathrm{~V}_{\mathrm{SE}}=124, \mathrm{~V}_{\mathrm{SC}}=122$. Similarly, BVA is the sequence of reverse virtual port number sequence. Therefore, the reverse route of $\mathrm{S}->\mathrm{D} \mathrm{BV}_{\mathrm{SD}}=111$. Bidirectional path represented by a vector address as Expression 1.

$$
\left(f_{1}, b_{1} ; f_{2}, b_{2} ; \cdots f_{n}, b_{n}\right)
$$

Where, $\left(f_{1} ; f_{2} ; \cdots f_{n}\right)$ represent forward vector address, and $\left(\mathrm{f}_{1} ; \mathrm{f}_{2} ; \cdots \mathrm{f}_{\mathrm{n}}\right)$ denotes backward vector address; $\mathrm{n}$ denotes the total number of hops.

After construction of vector address, coding of the vector address is setup using binary encoding. Thus, the representation of the vector address into binary form, $\mathrm{V}_{\mathrm{SD}}=123=\{1,2,3\} \rightarrow\{1,10,011\}$ that is, 110011. Each node encode virtual port numbers into binary code according to their degree. The virtual port numbers of All nodes from 1 to start coding, for example, the degree of B is equal to 4 , thence, the coding range of 001-100.

\section{CUTTING VECTOR ROUTING ALGORITHM}

After the vector coding, every node get route by cutting vector from packet. Fig. 4. shows the packet format of vector network.

Fig. 4. is Packet format of vector network.

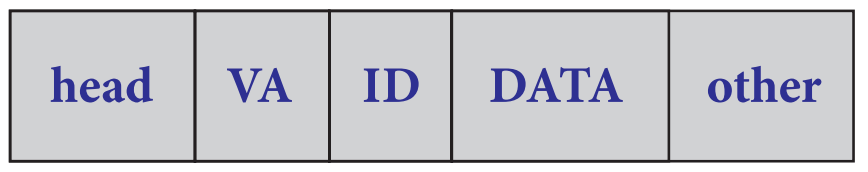

Fig. 4. An example of virtual port number were constructed

Where, head denotes head of packet, ID is increasing along with the packet forwarding and it used to indicate the freshness of the packet, like the same in AODV scheme. After a node receives a packet, firstly,separate the first component of VA and assigned to the variable sentto; secondly, remove the separated components from the VA field; thirdly, send packets to virtual port sentto. Fig. 5. show an example of cutting vector.

The steps of the algorithm are described as follows:

1. When first start algorithm, all nodes periodically broadcast ROUTE_PROBE to all neighbor nodes to establish a network topology.

2. Construction of the virtual port number(VPN) for all nodes based on network topology and encode VPN to vector address. 
3. Each nodes computes the length of the vector, and the minimun length of 10 vector paths are stored as set $\mathrm{S}$.

Fig. 5. is an example of cutting vector.

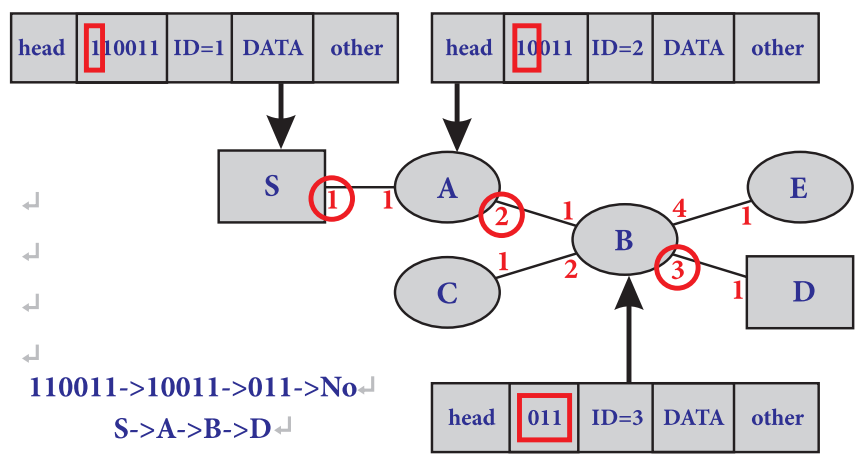

Fig. 5. An example of virtual port number were constructed

4. All nodes Periodically update the routing. During routing updating, the node in $S$ is preferentially chosen to be the next hop. Node sends data, after successfully updated routing. If $S=\varnothing$, goto 1 . Detail of the same algorithm in Algorithm 1.

\section{Algorithm 1 VNDR Algorithm:}

1. $\mathrm{N}=$ Total number of nodes

2. $\mathrm{j}=$ a sensor node in the network

3. j.VPN $=$ total number of virtual port

4. Vector $=$ virtual port encode as a binary vector

5. $s=$ the shortest path set

6. this = pointer to current node

7. $\operatorname{If}(s=\varnothing)$

8.

9. for $\mathrm{i}=1: \mathrm{N}$

10. Send ROUTE_PROBE to all neighbor nodes

11. $\mathrm{x}=$ list of all ROUTE_PROBE from neighbor

12. j.VPN $=$ Get the VPN of node

13. j.vector $=$ virtual port encode as a binary vector address

14. if(this.length(vector) $<$ length(i.vector))

15. if(s.Count $<10)$

16. add this.vector into $s$

17. else

18. Sent ROUTE_PROBE to a node in $\mathrm{s}$

19. if(receive REPLY_PROBE)

20. NEXT_HOP = cut vector(this.vector)

21. sent data to NEXT_HOP

22. else

23. goto 10

\section{SIMULATION AND RESULTS}

In this section, we present the simulation results using NS - 3. The proposed algorithm is compared with SPIN and DD. SPIN(Sensor Protocol for Information via Negotiation) is a data-centric adaptive routing communication protocol, and $\mathrm{DD}$ (Directed Diffusion) is a query-based routing algorithm, also is wireless sensor network protocol based on data related. We varied the total number of sensor nodes from 50 to 400 which are randomly deployed in a sensor field of A circular area with a radius of $50 \mathrm{~m}$. Wireless signal transmission model using the free space model, the propagation loss is calculated by the Formula 2.Furthermore, we uses the energy consumption model used in [1]. Sending and receiving energy consumpion as Formula 3 and Formula 4.

$$
\begin{gathered}
L_{p}=\left(\frac{\lambda}{4 \pi d}\right)^{2} \\
E_{t}=k \times E_{\text {elec }}+k \times E_{\text {amp }} \times d^{2} \\
E_{r}=k \times E_{\text {elec }}
\end{gathered}
$$

In Eq (2), (3) and (4), $L_{p}$ is the path energy loss, $d$ is the distance between sender and receiver, $\lambda$ is the wavelength of the signal, $E_{\text {elec }}$ is the cost of wireless transmitter and receiver, $E_{\text {amp }}$ is the spent of wireless transmission amplifier, $k$ is the packet length. In our experiment, set $E_{\text {elec }}=50 \mathrm{~nJ} / \mathrm{bit}, E_{\text {amp }}=100 \mathrm{pJ} / \mathrm{bit} \times \mathrm{m}^{2}$ and $k=10 b i t s$. Initially, all nodes in the network is powered by two battery-powered, and have a unified energy $5000 \mathrm{~J}$ (Because a battery can release $2500 \mathrm{~J}$ energy). Simulation parameters and values are listed in Tab2.

We compared VNDR scheme with SPIN and DD from the following aspects: route discovery cost, route signaling cost, average network energy consumption and average network lifetime.

Tab. 2. Simulation parameters and values.

\begin{tabular}{|c|c|}
\hline Parameters & Values \\
\hline Sensor field radiu & $100 \mathrm{~m}$ \\
\hline$E_{\text {elec }}$ & $50 \mathrm{~nJ} / \mathrm{bit}$ \\
\hline$E_{\text {amp }}$ & $100 \mathrm{pJ} / \mathrm{bit} \times \mathrm{m}^{2}$ \\
\hline$k$ & $10 \mathrm{bits}$ \\
\hline Initially energy per node & $5000 \mathrm{~J}$ \\
\hline $\mathrm{v}$ & $2 \mathrm{~m} / \mathrm{s}$ \\
\hline
\end{tabular}

THE ROUTE DISCOVERY COST

The Route Discovery Cost is used to estimate the cost of establishing a network topology consuming energy. Fig. 6. compares the per round route discovery cost for different network sizes.

As demonstrated in Fig. 6., spend less energy use the VNDR algorithm for establishing the network topology. In the network initialization phase, three schemes make use of flooding to discovery route. For small-scale network, VNDR, DD and SPIN energy consumption difference is not large, but from Fig. 6. can clearly see that the energy consumption of SPIN is twice than VNDR, and energy consumption of DD between VNDR and SPIN. In DD, each node needs to maintain a Interest Message List(IML) consuming more energy, therefore can not be used for large-scale networks and dynamic topology networks. In SPIN, each node should be carry out consultation by sending 
metadata before transmit the packet, this will result in large data transmission delay. In our proposed VNDR, each node uses vector cutting to achieve route discovery, which greatly reducing the overhead. As presented in Fig. 6. our proposed VNDR scheme has superior performance in route discovery cost.

Fig. 6. is Comparing the Per Round Route Discovery Cost for different network sizes.

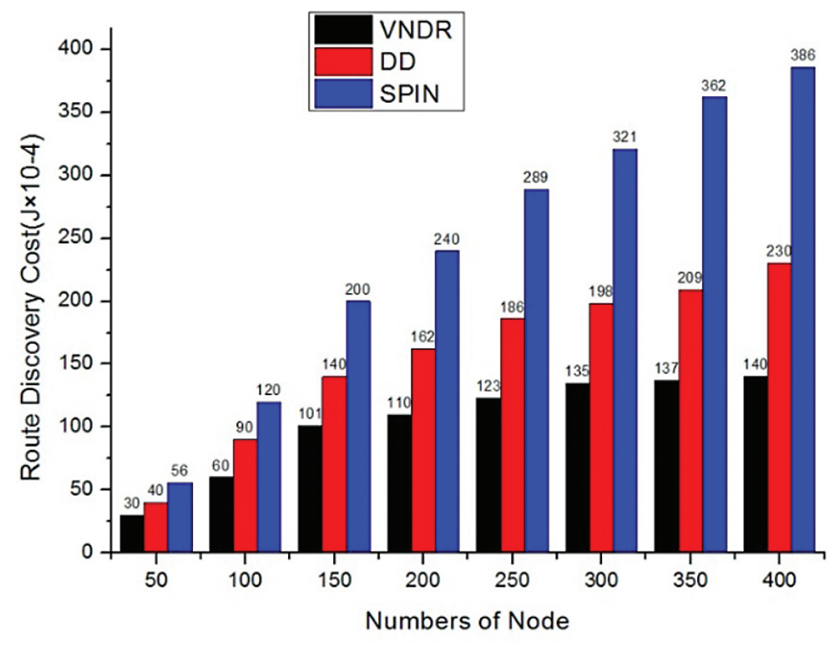

Fig. 6. Comparing the Per Round Route Discovery Cost for different network sizes

\section{THE ROUTE SIGNALING COST}

The Route Signaling Cost used to measure the proportion of the nerwork control signal and redundant data in the total amount of data, and it consists of two parts: signaling and overhead. The VNDR scheme employs Vector Address to control dynamic topology, corresponds to SPIN uses ADV packet for routing control which contains metadata and its own address, and DD make use of Gradient for topology control which consists of the transmission rate and transmission direction. As shown in Fig. 7., along with the network size grows, gradually increasing the signaling overhead. Moreover, our VNDR scheme demonstrated superior performance than SPIN and DD. Although VNDR to maintain the 10 shortest vector, since each vector address employ binary code to save a lot of storage space and control overhead.

\section{THE AVERAGE NETWORK ENERGY CONSUMPTION}

As shown in Fig. 8., spend less energy use the VNDR algorithm for establishing the network topology. With the number of nodes in the network increases, VNDR, SPIN and DD energy consumption are gradually increasing. When the number of nodes is small, VNDR, SPIN and DD energy consumption is very close. For example, when the number of nodes equal to $30, \mathrm{VNDR}$ and DD energy consumption is very close to, but SPIN energy consumption slightly higher. The VNDR scheme complete route discovery by the vector address binary coding, and this method effectively reduces the duty cycle of the control data, thereby reducing the energy consumption of route discovery. Similarly, DD by sending node interest to complete routing discovery. The node interest described by structure, which takes up more resources than binary vector address, thus, DD has more cost. Also, the SPIN wasting more energy by dropping packets when the packets is not required.

Fig. 7 is Comparing the Per Round Route Signaling Cost for different network sizes.

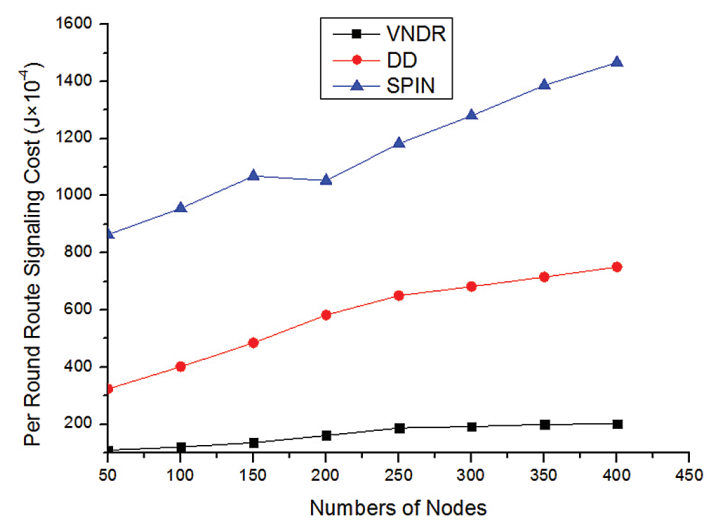

Fig. 7. Comparing the Per Round Route Signaling Cost for different network sizes

\section{THE AVERAGE NETWORK LIFETIME}

The average network lifetime used to measure the length of the network life, is the period of time from the network initialization to any node death due to the energy depletion. In our experiments, we use numbers of round to measure the average network lifetime. As presented in Fig. 8, with the increase in numbers of nodes, the numbers of rounds is in decreasing. Our VNDR algorithm with better performance on the number of rounds than other solutions. The VNDR network lifetime is foure times SPIN, and is DD two times. Using our VNDR scheme, effectively extending the life of network. This is mainly attributed to employing Virtual Port and vector addresses, that result in effectively reduce the control overhead, reduces the amount of data transmission, and prolong the network lifetime.

Fig. 8. is Comparing node average energy consumption for network working time.

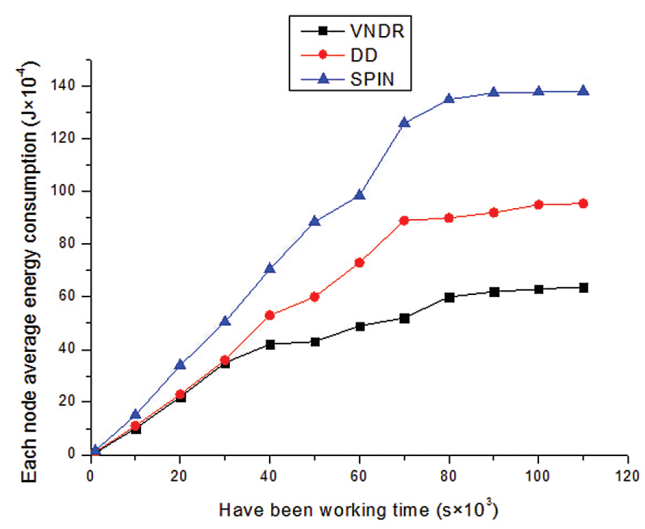

Fig. 8. Comparing node average energy consumption for network working time 
Fig.9 is Comparing the Number of Rounds for different network sizes.

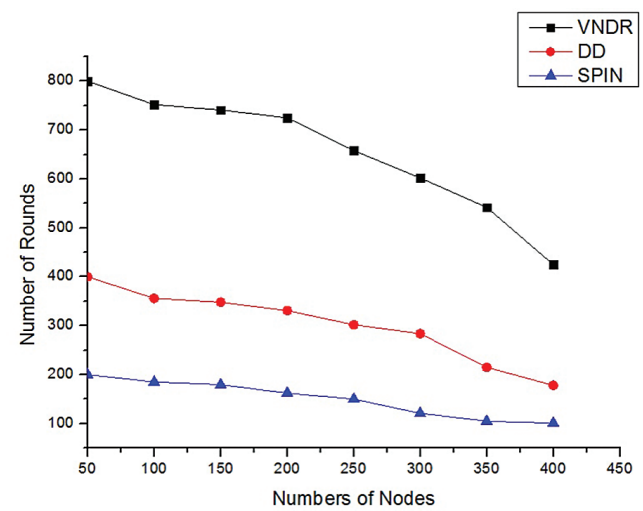

Fig. 9. Comparing the Number of Rounds for different network sizes

\section{CONCLUSION}

A new dynamic routes scheme VNDR is proposed for mobile WSN using vector address in this paper. Our VNDR scheme, first construct vector address based on virtual port number of node, and then embedded vector address in the packet. In data transfer phase, the next hop formed by cutting the vector algorithm. The simulation results revesls the VNDR reduced the control overhead and prolonged the network lifetime. In future, we will develop a test bed for VNDR scheme and hardware simulation.

\section{ACKNOWLEDGEMENTS}

This work was financially supported by National Natural Science Foundation Project, project number: U1636109, and National 863 Project, project number: 2007AA01Z203, and Hebei provincial science and technology plan special work projects, NO: 16236004D-8, and Project of 2016 Science and Technology Program of Zhangjiakou City, NO: 1611059B.

\section{REFERENCES}

1. Abdul Waheed Khan, Abdul Hanan Abdullah,Mohammad Abdur Razzaque,and Javed Iqbal Bangash. VGDRA: A Virtual Grid-Based Dynamic Routes Adjustment Scheme for Mobile Sink-Based Wireless Sensor Networks, IEEE SENSORS JOURNAL, VOL. 15, NO. 1, JANUARY 2015

2. Ravinder Kaura, Kamal Preet Singhb. An Efficient Multipath Dynamic Routing Protocol for Mobile WSNs, International Conference on Information and Communication Technologies (ICICT 2014),Procedia Computer Science 46 (2015) 1032-1040

3. Tapan Kumar Jain, Davinder Singh Saini, and Sunil Vidya Bhooshan.Lifetime Optimization of a Multiple Sink Wireless Sensor Network through Energy Balancing.Journal of Sensors Volume 2015, Article ID 921250, 6 pages
4. Jiliang Wang,Wei Dong,Zhichao Cao,and Yunhao Liu. On the Delay Performance in a Large-Scale Wireless Sensor Network: Measurement, Analysis, and Implications. IEEE/ ACM TRANSACTIONS ON NETWORKING,

5. Gabriel Mujica,Jorge Portilla,Teresa Riesgo. Performance evaluation of an AODV-based routing protocol implementation by using a novel in-field WSN diagnosis tool. Microprocessors and Microsystems 39 (2015) 920-938

6. B.Baranidharan, B.Santhi. DUCF: Distributed load balancing Unequal Clustering in wirelesssensor networks using Fuzzy approach. Applied Soft Computing 40 (2016) 495-506

7. Mohammad Hammoudeh, Robert Newman. Adaptive routing in wireless sensor networks: QoS optimisation for enhanced application performance. Information Fusion, Volume 22, March 2015, pp. 3-15

8. Fatemeh Mansourkiaie, Mohamed H. Ahmed. Per-Node Traffic Load in Cooperative Wireless Sensor Networks. IEEE COMMUNICATIONS LETTERS, VOL. 20, NO. 2, FEBRUARY 2016

9. Suraj Sharma and Sanjay Kumar Jena. Cluster based Multipath Routing Protocol for Wireless Sensor Networks. ACM SIGCOMM Computer Communication Review, Volume 45, Number 2, April 2015

10. Saeid Mottaghi, Mohammad Reza Zahabi. Optimizing LEACH clustering algorithm with mobile sink andrendezvous nodes. Int.J.Electron.Commun.(AEÜ) 69(2015)50-514

11. EtimadFadel,V.C.Gungor,LailaNassef,NadineAkkari,M. G.AbbasMalik,SuleimanAlmasri,IanF.Akyildiz.A survey on wireless sensor networks for smart grid. Computer Communications 71 (2015) 22-33

12. José J. Anaya, Edgar Talavera, Felipe Jiménez, Francisco Serradilla, José E. Naranjo. Vehicle to Vehicle GeoNetworking using Wireless Sensor Networks. Ad Hoc Networks 27 (2015) $133-146$

13. T.Hayes, F.H.Ali. Proactive Highly Ambulatory Sensor Routing (PHASeR) protocol for mobile wireless sensor networks. Pervasive and Mobile Computing 21 (2015) 47-61

14. Zhang Shuai, Liang, Man-Gui, Jiang Zhong-Yuan, Li HuiJia. Improved efficient static weighted routing strategy on twolayer complex networks, International Journal of Modern Physics C, 2015, 26(1).

15. An, Wenjuan, Liang, Mangui, Liu, He. An improved oneclass support vector machine classifier for outlier detection, Proceedings of the Institution of Mechanical Engineers, Part C: Journal of Mechanical Engineering Science, 2015, 229(3): 580-588. 
16. Jiang Zhong-Yuan, Liang Man-Gui, An Wen-Juan. Effects of efficient edge rewiring strategies on network transport efficiency, Physica A: Statistical Mechanics and Its Applications, 2014, 394: 379-385.

17. Zhang Shuai, Liang Man-Gui, Jiang Zhong-Yuan, Wu JiaJing. Effective strategy of adding links for improving network transport efficiency on complex networks, International Journal of Modern Physics C, 2014, 25(6).

18. Liu He, Liang Mangui. Privacy-preserving registration protocol for mobile network, International Journal of mmunication Systems, 2014, 27(10): 1653-1671.

19. Wang Shujuan, Liang Mangui. An Efficient Multicast Strategy for On-Demand Data Dissemination in Wireless Networks, Journal of Internet Technology, 2014, 15(4): 557-564.

20. Zhang Shuai, Liang Man-Gui, Li Hui-Jia. Method to enhance traffic capacity for two-layer complex networks, Canadian Journal of Physics, 2014, 92(12):1599-1605.

21. Jiang Zhong-Yuan, Liang Man-Gui, Wu Jia-Jing. Dynamic Source Routing Strategy for Two-Level Flows on Scale-Free Networks, PLos One, 2013, 8(12).

\section{CONTACT WITH THE AUTHOR}

Liang Mangui, Ph. D.

e-mail: mgliang@bjtu.edu.cn tel: 13801166970

Institute of Information Science Beijing Jiaotong University Beijing 100044

China 